\title{
Extraction of Pure-electronic Transition Frequency and Chromophor Polymorphism from Diffuse Vibronic Spectra
}

\author{
Vitaly Antonovich Tolkachev*, Alexander Pavlovich Blokhin \\ Institute of Physics, National Academy of Sciences of Belarus, Minsk, Belarus \\ Email address: \\ tolkachev@imaph.bas-net.by (V. A. Tolkachev),1sfm@imaph.bas-net.by (A. P. Blokhin) \\ ${ }^{*}$ Corresponding author
}

\section{To cite this article:}

Vitaly AntonovichTolkachev, Alexander Pavlovich Blokhin. Extraction of Pure-electronic Transition Frequency and Chromophor Polymorphism from Diffuse Vibronic Spectra. Science Journal of Analytical Chemistry. Vol. 7, No. 4, 2019, pp. 76-82.

doi: $10.11648 /$ j.sjac.20190704.11

Received: July 19, 2019; Accepted: September 6, 2019; Published: September 21, 2019

\begin{abstract}
Background: The adiabatic approach and thermal population of starting state sublevels in vibronic transition at non degenerate combining states of molecular chromophores open ways to calculate pure-electronic transition frequency (combining states gap) individually from diffuse absorption or emission spectra. Results: Experimental data and the theory show, that the model fits to homogene chromophores at room and not low temperatures to escape degeneration and inhomogeneity. Side result of the approach is possibility to view inhomogeneity of chromophores or solvent site inhomogeneity. Conclusions: The approach is applied to vibronic spectra of molecular systems: molecules in different aggregate states, molecular crystals, color and F-centers, films and quantum dots. The trouble with the procedure is using wings of spectra, where the errors can be introduced by overlapping of impurities spectra and even by measurement inaccuracy.
\end{abstract}

Keywords: Diffuse Vibronic Spectrum, Pure-electronic Transition, Zero-Phonon Frequency, Chromophore Homogeneity, Quantum Dots Homogeneity, Molecular Crystals, F-centers and Color Centers

\section{Introduction}

It was shown recently [1-4], that frequency of purely electronic, $0-0$-transitions could be determined from continuous diffuse crossection spectra of only absorption or only emission (fluorescence, phosphorescence) for homomorphic chromophors (molecules, molecular complex, etc.) at equilibrium distribution of population upon initial-state sublevels. But the base of the procedure were rather complicated and discussed fluently. Though the idea has been confirmed by numerous experimental data [1-4] it needs to be revised, summed and commented.

$$
\left|D_{i, j, k}\right|^{2}=\left|<\Psi_{i}\left(x_{i}, q_{k}\right)\right| \vec{M}\left(q_{k}\right)\left|\Psi_{j}^{*}\left(x_{j}, q_{k}\right)>\right|^{2}=\left|<\Psi_{j}\left(x_{j}, q_{k}\right)\right| \vec{M}\left(q_{k}\right)\left|\Psi_{i}^{*}\left(x_{i}, q_{k}\right)>\right|^{2}
$$

where $\vec{M}\left(q_{k}\right)$ are dipole moment of rigid nuclei structure. The value of dipole moment

\section{Theory}

\subsection{General Conception}

Optical electron-dipole transition proceeds as transformation of electronic component in ensemble "electrons+nuclei" because mobility of electronic component is substantially higher. The model with functions of starting $\Psi_{i}\left(x_{i}, q_{k}\right)$ and finishing $\Psi_{j}\left(x_{j}, q_{k}\right)$ states, where $x_{i, j}$ and $q_{k}$ are generalized coordinate of electrons and nuclei, when in elementary state of the system dipole transition crossections are reversible, gives 


$$
\left|D_{i, i, k}\right|^{2}=\left|<\Psi_{i}\left(x_{i}, q_{k}\right)\right| \vec{M}\left(q_{k}\right)\left|\Psi_{i}^{*}\left(x_{i}, q_{k}\right)>\right|^{2}
$$

Corresponds to pure-electronic transition $\left(h v_{0}\right)$, which at low temperature limit is 0-0-transition. In the scope of combining start $\left(\mathrm{E}_{1}\right)$ and finish $\left(\mathrm{E}_{2}\right)$ state the energies for depletion of transition quantum on $h \Delta v=h\left|v-v_{0}\right|$ about $h v_{0}$ the crossections are equal and proportional to

$$
\left|D\left(E_{1}, E_{2}\right)\right|^{2}=\left|D\left(E_{2}, E_{1}\right)\right|^{2}=\left|D\left(E_{1}, E_{1} \pm h \Delta v\right)\right|^{2}=\left|D\left(E_{1} \pm h \Delta v, E_{1}\right)\right|^{2},
$$

$\left(E_{1}, E_{2}>0\right)$. So the crossection for transition $E_{1} \rightarrow E_{1}+h \Delta v$ in Stokes region $\left(v>v_{0}\right)$ at thermal equilibrium onsublevels of ground state is proportional to

$$
\frac{\varepsilon\left(v_{0}+\Delta v\right)}{v_{0}+\Delta v}=\mathrm{const} \cdot \int_{0}^{\infty} e^{-E_{1} / k T} \cdot g\left(E_{1}\right) \cdot g\left(E_{1}+h \Delta v\right) \cdot\left|D\left(E_{1}, E_{1}+h \Delta v\right)\right|^{2} d E_{1},
$$

where $\mathrm{g}(\mathrm{E})$ and $\mathrm{g}(\mathrm{E}+\mathrm{h} \Delta v)$ are the statistical weights of combining states, $\varepsilon$ - absorption coefficient. For mirror to (4) transitions $\left(E_{1}+h \Delta v \rightarrow E_{1}\right)$ with energy conservation one obtains

$$
\frac{\varepsilon\left(v_{0}-\Delta v\right)}{v_{0}-\Delta v}=e^{-h \Delta v / k T} \cdot \frac{\varepsilon\left(v_{0}+\Delta v\right)}{v_{0}+\Delta v}-c o n s t \cdot e^{-h \Delta v / k T} \int_{0}^{h \Delta v} e^{-E_{1} / k T} \cdot g\left(E_{1}+h \Delta v\right) \cdot g\left(E_{1}\right) \cdot\left|D\left(E_{1}+h \Delta v, E_{1}\right)\right|^{2} d E_{1}
$$

Multiplying (5) by $\exp (\mathrm{h} \Delta v / 2 \mathrm{kT})$ and taking into account that at $\Delta v \leq 0$ the integral in (5) disappears we obtain

$$
f^{a}\left(v_{0},\left|v-v_{0}\right|\right)=\frac{\varepsilon\left(v_{0}-\Delta v\right)}{v_{0}-\Delta v} \cdot e^{h \Delta v / 2 k T}=e^{-h \Delta v / 2 k T} \cdot \frac{\varepsilon\left(v_{0}+\Delta v\right)}{v_{0}+\Delta v},
$$

the relation, which is symmetric about $v_{0}$. By similar way for emission $E_{2} \rightarrow E_{2}+h \Delta v$ with $v \leq v_{0}$ for emission intensity $I$ one obtains

$$
f^{e}\left(v_{0},\left|v-v_{0}\right|\right)=\frac{I\left(v_{0}+\Delta v\right)}{\left(v_{0}+\Delta v\right)^{4}} \cdot e^{-h \Delta v / 2 k T}=\frac{I\left(v_{0}-\Delta v\right)}{\left(v_{0}-\Delta v\right)^{4}} \cdot e^{h \Delta v / 2 k T}
$$

As relations (6) and (7) are symmetric about $v_{0}$, so at that frequency functions $f^{a}\left(v_{0}, \Delta v^{2}\right)$ and $f^{e}\left(v_{0}, \Delta v^{2}\right)$ have extremum. The relative value of the integral in (5) depends on temperature by population of energy interval $[0, \mathrm{~h} \Delta v]$. Whentemperature rise the relative input of the interval population and the value of the integralin (5) drops, reducing asymmetry which is introduced bythe integral in (5). So in region of $h \Delta v<k T$ one can observe approximated symmetry of f-functions about $v_{0}$. The same is for emission. That behaviorwas observed on experimental data [1-4]. As the relationships (6) and (7) operate in Stokes regions, it means, that

$$
\begin{aligned}
& \frac{\varepsilon\left(v_{0}+\Delta v\right)}{v_{0}+\Delta v} \cdot e^{-h \Delta v / 2 k T}=f^{a}\left(v_{0}, \Delta v^{2}\right) ; v \geq v_{0} \\
& \frac{I\left(v_{0}-\Delta v\right)}{\left(v_{0}-\Delta v\right)^{4}} \cdot e^{h \Delta v / 2 k T}=f^{e}\left(v_{0}, \Delta v^{2}\right) ; v \leq v_{0}
\end{aligned}
$$

The practically more useful is logarithmic form, as applied in [1]-[4]:

$$
\ln \frac{\varepsilon_{a}(v)}{v}-\frac{h v}{2 k T}=\varphi^{a}\left(\left|v-v_{0}\right|\right), v \geq v_{0}
$$

$$
\ln \frac{I(v)}{v^{4}}+\frac{h v}{2 k T}=\varphi^{e}\left(\left|v-v_{0}\right|\right), v \leq v_{0},
$$

where $\varphi$ are normalized to interval $[0,1]$, though more sensitive and match to spectra order forms are nonlogarithmic.

One can see, that the method allows to calculate FrankCondon, vertical frequency $v_{0}$, where the finish state is a state of new configuration of electrons, but former, starting state of slow, nuclei system. The $v_{0}$ achieved from absorption spectra and the one for excited state differ as the finish structures of slow, nuclei and the new electronic systems after transitions relax to match in between and medium.

\subsection{Degeneration}

Many examples of molecular systems from molecular vapor to F-centers were considered in the approach $[1-4,9]$. The laser dye solutions as a rule have homogeneous chromophors and F-centers just opposite, what is vivid. The deviation from homogeneity at room and higher temperatures is structure or site inhomogeneity. At low temperatures the other mechanismsare added. Accepted as basic the condition (1) of 
optical dipole transition reversibility on the elementary level is correct for non degenerate systems. The systems are, for examples, polyatomic molecules, where at usual and high temperatures there are many intramolecular mechanisms which destroy degeneration. The role and influence of the mechanisms rise up with temperature and drop when the temperature drops. Interaction of degenerate states in optical transition shows complicated effects, which are controversial to the reversibility on molecular elementary level $([5,6]$ e. a.). At temperatures in dozen degrees Kelvin the extremum at $v_{0}$ is as a rule not observed at all. The reasons of deviation from obtained above relations at low temperatures are not only degeneration, but cell site and chromophore polymorphism

because of slow relaxation of states and the other mechanisms.

\subsection{Average Energies of Combining States}

The spectral selectivity of vibronic transitions is reflected in statistical characteristics. It was shown [7], that the dependence from temperature of vibronic absorption spectra, when the transition probability depends on temperature by population Boltzmann factor, is connected to the average energies of excited $\bar{E}_{a}^{*}(v)$ and of all ground state molecules $\bar{E}$ in vapor state by relation

$$
-\partial \ln (\varepsilon(v)) / \partial(1 / k T)=\bar{E}_{a}^{*}(v)-\bar{E}-h v
$$

From (10) and (12) it follows

$$
-\partial \ln f^{a} / \partial(1 / k T)=h v / 2-\partial \ln (\varepsilon(v)) / \partial(1 / k T)=\left(\bar{E}_{a}^{*}+\left(\bar{E}_{a}^{*}-h v\right)-2 \bar{E}\right) / 2
$$

The relation (13) is symmetrical about $v_{0}$ too. Besides, one can see, that extremum of (13) is extremum of the average energies sum of states combining in optical transitions [3]. Like above, for emission by relation

$$
-\partial \ln (I(v)) / \partial(1 / k T)=\bar{E}_{e}^{*}(v)-\bar{E}^{*}
$$

with $\bar{E}_{e}^{*}(v)$ as the average energy of excited molecules emitting photons $h v$ and the average energy of all excited molecules $\bar{E}^{*}[8]$ and (11) one obtains

$$
-\partial \ln f^{e} / \partial(1 / k T)=-h v / 2-\partial \ln I(v) / \partial(1 / k T)=\left(\bar{E}_{e}^{*}+\left(\bar{E}_{e}^{*}-h v\right)-2 \bar{E}^{*}\right) / 2
$$

As for absorption and emission the expressions (13) and (15) are symmetrical about $v_{0}$ so $v_{0}$ corresponds to minimal sum of combining states average energies.

\subsection{Mixture of Chromophors}

One of main shortage of the methods is basing on longwave in absorption and shortwave in emission low intensity wings of spectra. Overlapping the wings by other spectra adds problems. Overlapping spectra of $n$ kinds of chromophore modifies the relations (8) and (9) to

$$
\left(\sum_{n} \sigma_{n}(v)\right) \cdot \exp (-h v /(2 k T)) / v=\sum_{n} f_{n}\left(\left|v-v_{n 0}\right|\right)=f_{\text {gen }}^{a}
$$

for absorption and

$$
\left(\sum_{n} I_{n}(v)\right) \cdot \exp (h v /(2 k T)) / v^{4}=\sum_{n} f_{n}^{e}\left(\left|v-v_{i 0}\right|\right)=f_{g e n}^{e}
$$

for emission.

The extremum will smear because displacement and overlapping of particular extremums at different $v_{n 0}$ and determination of separate pure electronic transition is impossible. But the disappearance of extremum is a qualitative note of chromophore inhomogeneity [4, 9] and some will be added below.

When the spectra of different vibronic transitions overlap as for example in molecular UV-Vis absorption, their temperature dependence can help [3]. As the antistokes regions of spectra are more sensitive to temperature so their location indicate region of extremum.

\section{Approaches}

The results of above considered model are tested for the rather extensive types of electronic transitions [1-4] in molecular vapors, solutions, color centers, including F-centers. In following the new examples of the approach are demonstrated.

\subsection{Molecular Crystals}

It was not considered application the method to molecular crystals. The orientation of molecules in molecular crystals is very high [1-4]. But elementary cell in molecular crystal may consist from identical but differently oriented molecules, so 
the spectrum would appear as completed from different, inhomogeneous chromophore nature. The particular inputs to spectrum depend on the cell structure and on the direction of light wave polarization. Figure 1 shows the absorption

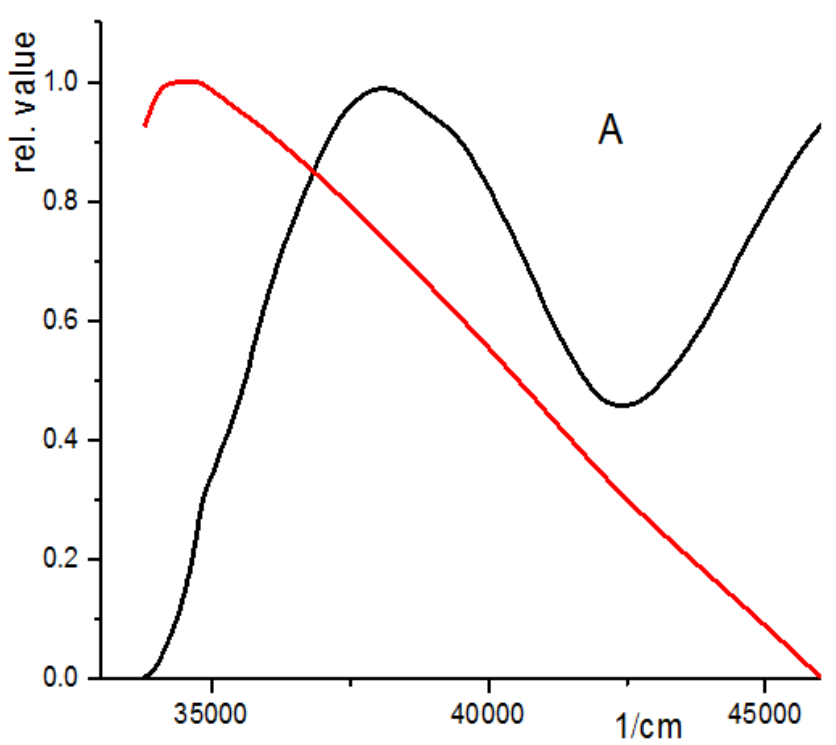
Figure 1. Absorption spectra of 1.3-dimethyluracil: A-
0-0-transition (35354 $\left.\mathrm{cm}^{-1}\right)$ found at jet-cooling [12].

Figure 2 gives the absorption spectra of uracil crystal at main and orthogonal optical axes of polarization. Here extremum is absent, indicating polymorphism of absorbing chromophors. Like shown in figure 2 the absorption spectra for polarizations $\| \mathrm{a}$ and $\| \mathrm{c}$ from [10] give polymorphism too. As it is known, uracil polymorphism is based on high lability of protons in the molecule. For the vapor state that polymorphism also found too [2]. The spike on $\varphi^{a}$ in figure $1 \mathrm{~A}$ at $34150 \mathrm{~cm}^{-1}$ possibly notes single prevailing orientation of uracil chromophore. The spikes like that, for example, are observed by authors in solutions of some dyes ( $\mathrm{Rh} 4 \mathrm{C}$ in

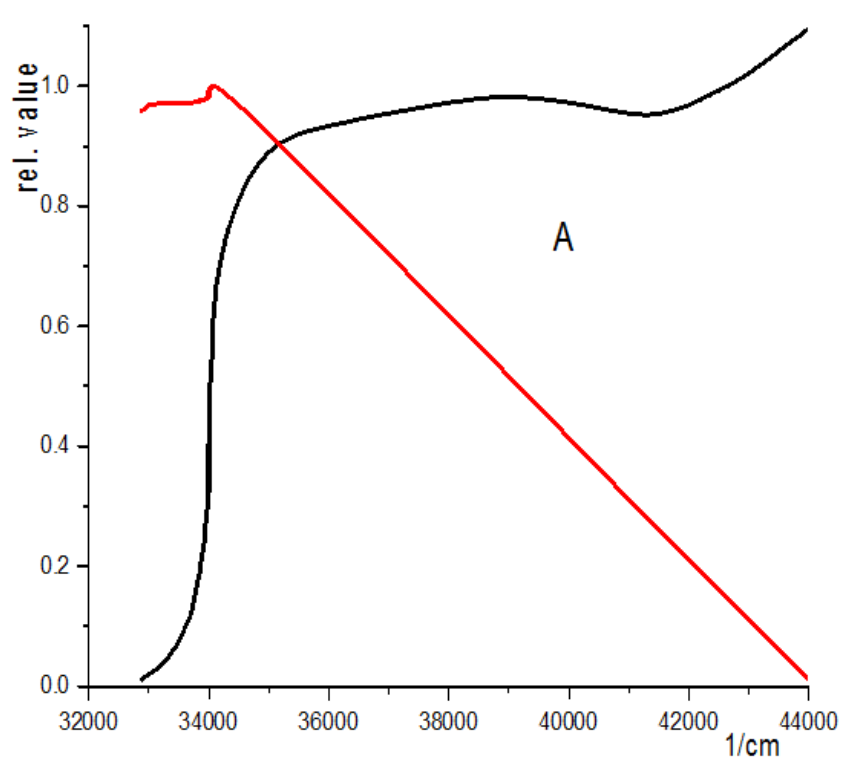

spectra and $\varphi$-functions of crystal and vapor of 1.3dimethyluracil. In both of aggregate states the absorbing structures are homomorphic, monochromoforic.

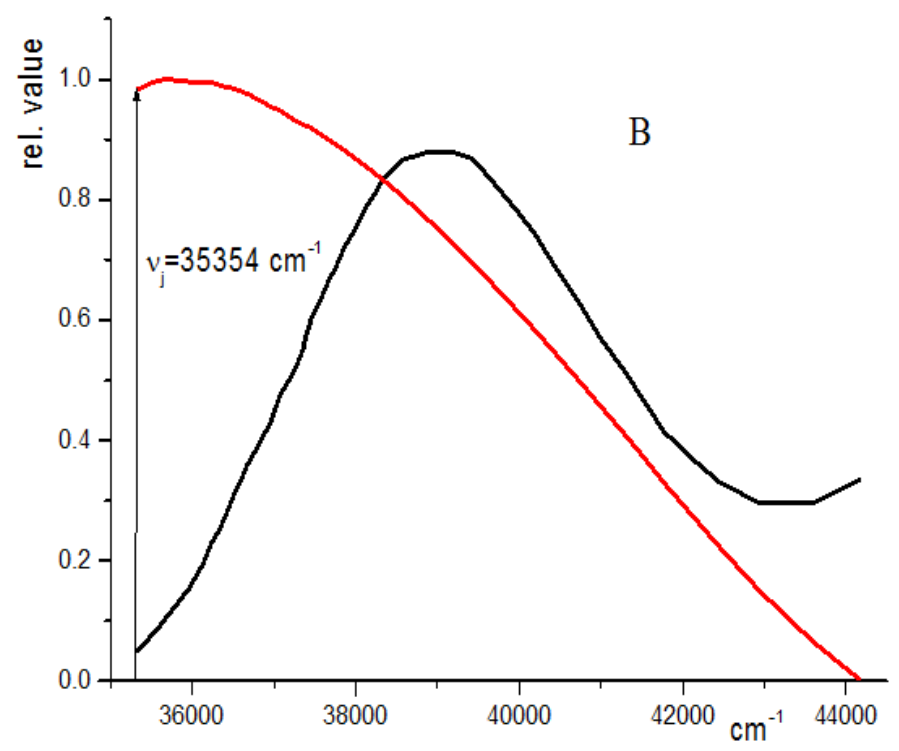

ethanol, BBO in ethanol and dioxane a. o.). By this method were analyzed the absorption spectra of thymines and uracils crystals from [8] and was found, that predominantly the homogeneous chromophors are of crystals 1.3-dimethyluracil, 1.3-dimethylthimine, monohydrate thymine, calciumtimidilate; inhomogeneous are chromophors of 2ethyl-5-methyluracil, uridine-5'-calciumphosphate, 1methylthymine. As noted above, reason may be proton migration or different orientation of molecules in elementary cell of the crystals.

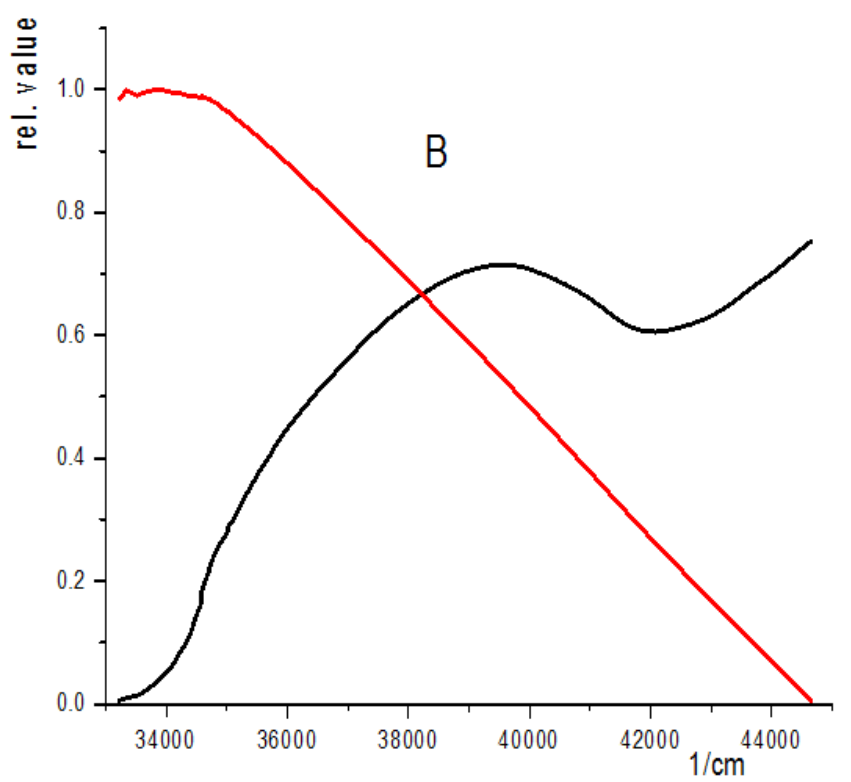

Figure 2. Absorption spectra of uracil crystals at room temperature for polarization along main optical axis $Z^{\prime}$ (A) and orthogonal X' (B) [10] and the corresponding $\varphi$-functions. 


\subsection{Quantum Dots}

There are some interesting examples of definition of quantum dots electronic transition by this method. Best fit as it will be seen below is for graphene-based quantum dots (GQD). There are two fundamental approaches to produce GQD: "top-down" and "bottom-up" approaches. The "topdown" approach is most popular and based on fragmentation of huge graphene block into small particle with following chemical modification. The rout "bottom-up" is synthesis of GQDs from molecular fragments of lower dimension. By chemical doping the GQDs of required properties, even luminescent, can be obtained. From much forms GQDs two luminescent forms of the GQD obtained by methods "bottom-up" and "top-down" were selected to the study. The emission spectra and $\varphi$-functions of the GQDs are shown in figure 3.
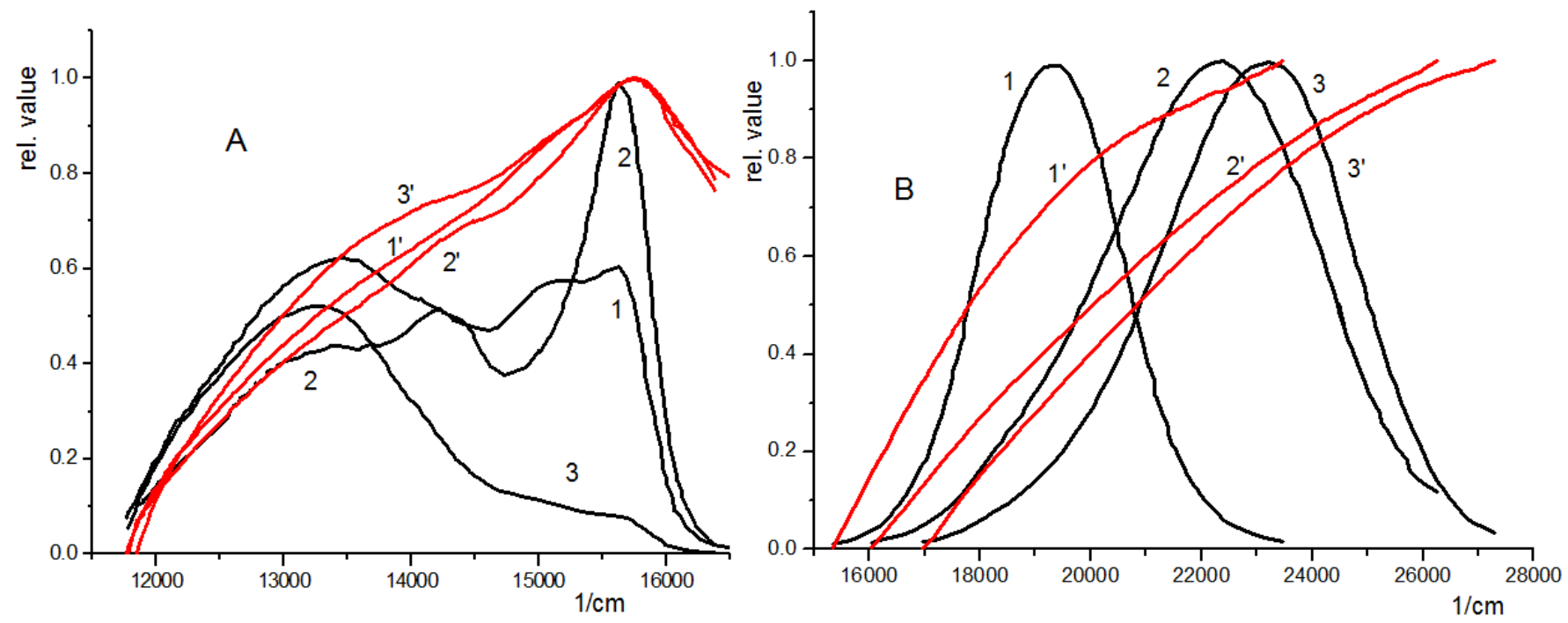

Figure 3. Emission spectra of GQD's: $A$ - C132, obtained by method "bottom-up": $1-\lambda_{e x}=337 \mathrm{~nm}, 2-\lambda_{e x}=479 \mathrm{~nm}$, 3- $\lambda_{e x}=532 \mathrm{~nm}$ [13, 14]; and (B) by "topdown" method: 1- non modified oxido-GQDs, 2- previous ones doped by aminogrups $m$-GQDs, 3- obtained from m-GQDs by reduction, $r$-GQDs [15]; 1'-3'the corresponding $\varphi$-functions.

The homogeneity of shortwave emitting chromophore of $\mathrm{C} 132$ is very high for all emission spectra excited by light of different wavelengths. The lower excited emitting level is about $15760 \mathrm{~cm}^{-1}$ over the ground state. At about $14000 \mathrm{~cm}^{-1}$ there are next overlapped transition, which probably may be determined by variation of temperature method. The found homogeneity of longwave C132 chromophore by $\varphi$-functions is supported by reliably established in [13] homogeneity of its structure. Just opposite are results on figure 3B. All here shown GQD ensembles are mostly inhomogeneous. It is consistent with the production technology.

On figure 4 by the developed method evolution of diamond nanoparticle fluorescence spectra after oxidation and annealing [16] are analyzed. To obtain homogeneous concentration of NV-centers the nanoparticles were oxidized to depart unnecessary substitutuents and then annealed. It is seen, that the oxidized particles without annealing are mostly inhomogeneous. The annealing increases the homogeneity with temperature and at temperatures $800^{\circ} \mathrm{C}-900^{\circ} \mathrm{C}$ attains the homogeneity. The pure electronic frequency is indicated at about $17390 \mathrm{~cm}^{-1}$ and is seen for all samples, but mostly sharp extremum is for homogeneous samples. The obtained transition frequency coincides with the transition of known $\mathrm{NV}^{\circ}$ emission centers from state of symmetry ${ }^{2} \mathrm{~A}_{1}$ to ground state $^{2} \mathrm{E}[17,18]$.

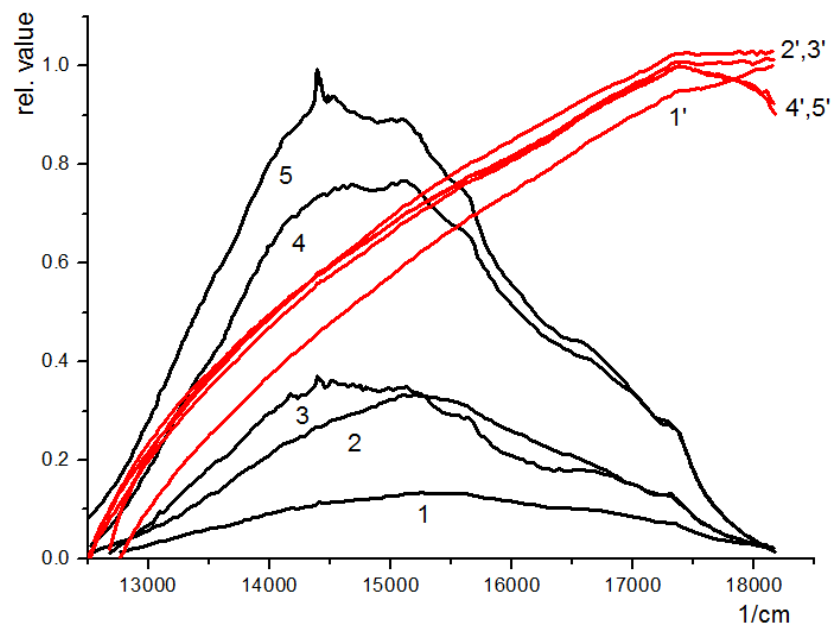

Figure 4. Fluorescence spectra of diamond nanoparticles, obtained by oxidation in air with no annealing (1) and after annealing at temperatures 2$650^{\circ} \mathrm{C}, 3-700^{\circ} \mathrm{C}, 4-800^{\circ} \mathrm{C}, 5-900^{\circ} \mathrm{C}[16] ; 1^{\prime}-5^{\prime}-$ relative $\varphi^{a}$-functions.

\subsection{Dichroism}

Figure 5 shows examples of correlation the dichroism spectra and of $\varphi$-functions. The dichroism of 9methyladenine in stretched films PVA on figure 5A shows best correlation of dichroism and $\varphi$-function in longwave 
wing of absorption spectra, though the spectrum is in some measure inhomogeneous. In figure $5 \mathrm{~B}$, as shown by the $\varphi$ function spectra, both forms of the proteins in anisotropic

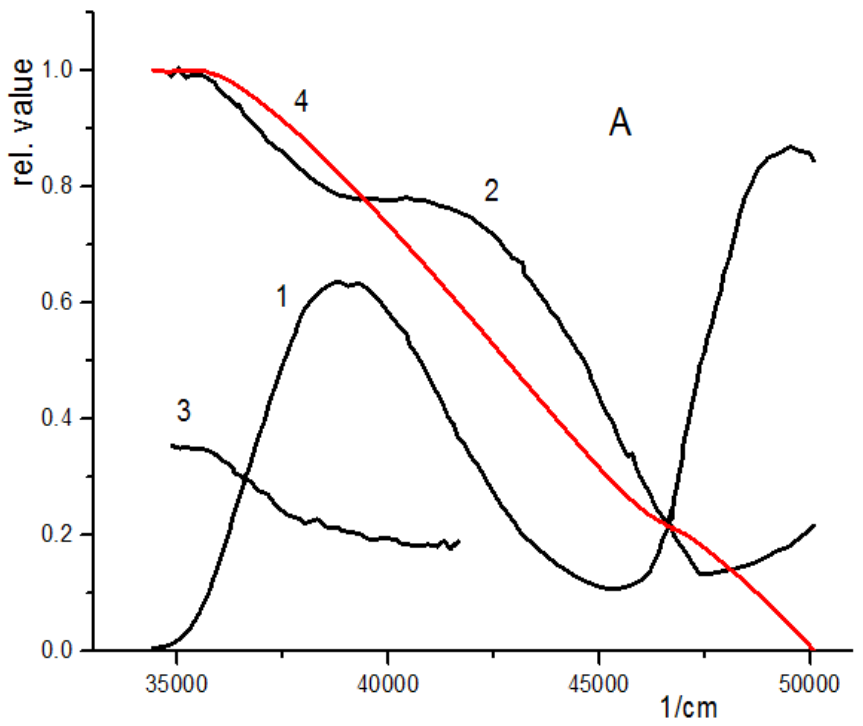

polyacrylamide gel have rather low inhomogeneity, supported by dichroism spectra, though chromophors of the proteins have complicated orientation [19].

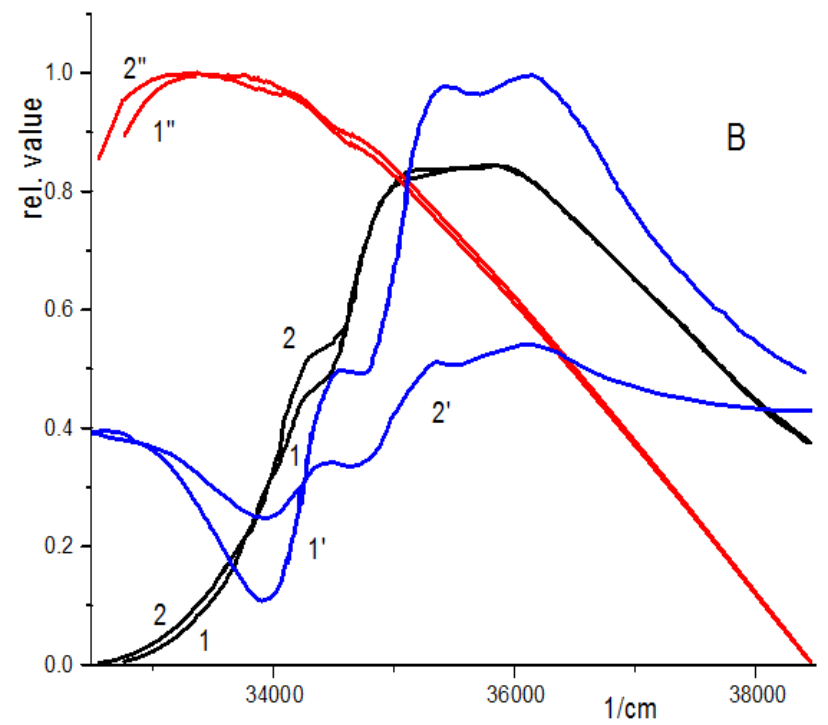

Figure 5. Absorption and dichroism spectra. A -9-methyladenine in PVA film: 1 - absorption, 2 - linear dichroism; 3 - fluorescence anisotropy in EPA at $80 \mathrm{~K}$ [20], 4 - $\varphi^{a}$-function; B- proteins bovine $\gamma I I-$ and $\gamma$ IVa-crystallins in anisotropic gel: 1, 2 - absorption spectra, 1', 2'- linear dichroism [19], 1", 2" - $\varphi^{a}$ functions.

\subsection{Light Quenching}

Figure 6 shows the correlation of fluorescence, relative yield of light quenching [21] and $\varphi^{e}$-function 1.1'.4.4'tetraphenylbutadiene (TPB) spectra. The quenching goes by $S_{1} \stackrel{h v_{q}}{\longrightarrow} S_{0}$ mechanism. That kind of quenching must drop when $h v_{q}>h v_{0}$ as one can see here. Such dependence was observed in [21] for solutions of POPOP in hexane and benzene too, but TPB is demonstrated here, because the fluorescence spectra of POPOP are not diffuse.

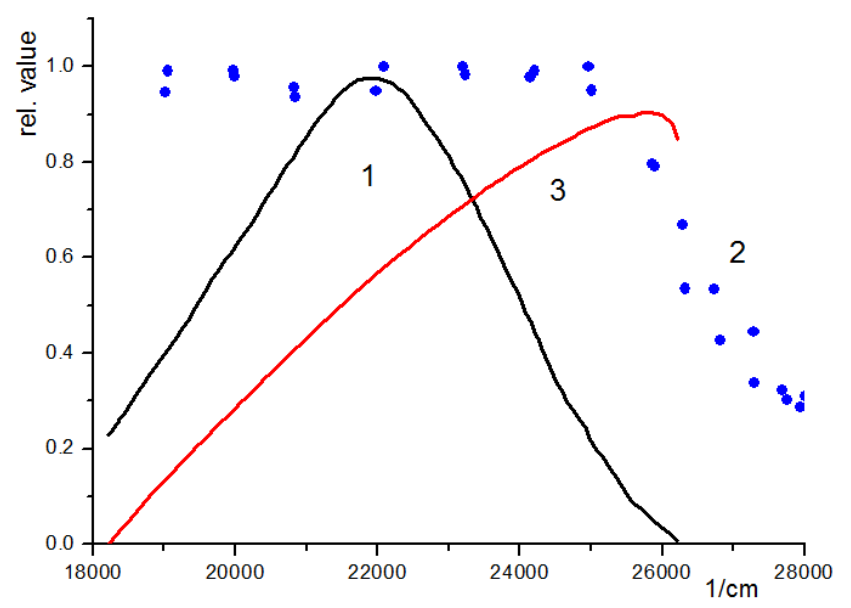

Figure 6. Solution of TPB in hexane. Fluorescence (1) and relative yield of fluorescence quenching by light (2) spectra [21], $\varphi^{e}$-function (3).

\section{Conclusions}

Obtained by this method $v_{0}$ of molecular vapors and $0-0$ transition found by jet-cool method differ less then 200-300 $\mathrm{cm}^{-1}(0.02-0.04 \mathrm{eV})$ though the temperatures differ on hundreds of degrees, but the differences between diffuse spectra maxima and $v_{0}$ are about $0.1-0.4 \mathrm{eV}[1,2]$. The analyzed above and tested in different situations method is useful not only by better location of electronic transition, but by possibility to determine the transitions from absorption or emission spectra separately, as the sample structure permits. A side capability is appeared to see the inhomogeneity of forming spectrum chromophors and some time to do the conclusion on chromophors media inhomogeneity. The approach is not free from limitation and shortcoming. The main vivid lacks are not determined levels of negative degeneration influence, of low temperature effect. The wings of spectra to use are uncomfortable for measurements: besides of different chromophors the errors can be introduced by impurities and even by measurements.

\section{Acknowledgements}

The authors thank the Belarusian Republican Foundation for Fundamental Research for supporting the work (Project F18UKA-030).

\section{References}

[1] V. A. Tolkachev "Determination of 0-0-Transition Frequencies from Diffuse Vibronic Spectra". Journal of Applied Spectroscopy, 84, 2017, 668-673.

[2] V. A. Tolkachev "Position of 0-0-Transition Frequency in Diffuse Vibronic Spectrum". Doklady Natsyonal'noi Akademii Nauk Belarusi, 61, 2017, 50-55 (rus.). 
[3] V. A. Tolkachev "Average Energies of Combining States and Purely Electronic Transition Frequencies in Vibronic Spectra". Journal of Applied Spectroscopy, 85, 2018, 845-849.

[4] V. . Tolkachev "Manifestation of Molecular Chromophore Polymorphism in Diffuse Vibronic Spectra". Journal of Applied Spectroscopy, 86, 2019, 504-507.

[5] C. J. Chen and R. Wiesendanger "Perturbation Theory of Exchange Interaction". Physical Review B, 74, 2006, 113102.

[6] V. A. Tolkachev "Signs of Tunneling of $(n, \pi)$ Excitation in Fluorescence of cis-Azoalkanes". Journal of Applied Spectroscopy, 81, 2014, 688-690.

[7] V. Tolkachev "Dependence of Excitation Effective Energy of Vapor State Molecules on Exciting Light Frequency". Izvestiya. Akademii Nauk SSSR, ser. fiz., 27, 1963, 584-587 (rus.).

[8] V. A. Tolkachev and N. A. Borisevich "Average Energy of Fluorescing Molecules in Rarefied Vapors". Optika i Spektroskopiya, 15, 1963, 306-309. (rus.).

[9] V. A. Tolkachev "Manifestation of Molecular Chromophore Polymorphism in Diffuse Vibronic Spectra". Journal of Applied Spectroscopy, 85, 2018, 220-224.

[10] M. Tanaka and J. Tanaka "Electronic Absorption Spectra of Crystals of Thymine, Uracyl, and Their Derivatives". Bulletin of the Chemical Society of Japan, 44, 1979, 938-944.

[11] M. J. Novak, K. Szczepaniak, A. Barski and D. Shugar "Spectroscopic Studies on Vapour Phase Tautomerism of Natural Bases Found in Nucleic Acids". Z. Naturforsch., 33c, 1978, 876-883.

[12] Y. Tsuchia, T. Tamura, M. Fujii and M. Ito "Keto-Enol Tautomer of Uracil and Thymine". J. Phys. Chem., 92, 1988, $1760-1765$.

[13] X. Yan, X. Cui andL. Li "Synthesis of Large, Stable Colloidal
Graphene Quantum Dots with Tunable Size". J. Am. Chem. Soc., 132, 2010, 5944-5945.

[14] H. Riesen, Ch. Wieber and S. Schumacher "Optical Spectroscopy of Graphene Quantum Dots: The Case of C132". J. Phys. Chem. A, 118, 2014, 5189-5195.

[15] S. Zhu, J. Zhang, S. Tang, C. Qiao, L. Wang, H. Wang, X. Liu, B. Li, N. Yu, X. Wang, H. Sun and B. Yang "Photoluminescence Mechanism in Graphene Quantum Dots: Quantum Confinement Effect and Surface/Edge State". Adv. Funct. Mater., 22, 2012, 4732-4740.

[16] X. Song, G. Wang, X. Liu, F. Feng, J. Wang, L. Lou andW. Zhu "Generation of Nitrogen-Vacancy Color Center in Nanodiamonds by High Temperature Annealing". Appl. Phys. Lett., 102, 2013, 133109.

[17] G. Davies "The Jahn-Teller Effect and Vibronic Coupling at Deep Levels in Diamond". J. Phys. C Solid State Phys., 12, 1979, 2551-2556.

[18] S. Felton, A. M. Edmonds, M. E. Nevton, P. M. Martineau, D. Fisher and D. J. Twitchen "Electron Paramagnetic Resonance Studies of the Neutral Nitrogen Vacancy in Diamond". Phys. Rev. B, 77, 2008, 081201.

[19] M. Bloemendal, J. A. M. Leunissen, H. Amerogen and R. Grondelle "Average Orientation of Aromatic Residues in Proteins Determined from Linear Dichroism Spectroscopy". A Comparison of Results on Bovine7-Crystallins with X-ray Data". J. Mol. Biol., 216, 1990, 181-186.

[20] A. Holmen, A. Broo, B. Albinsson and B. Norden "Assignment of Electronic Transition Moment Directions of Adenine from Linear Dichroism Measurements". J. Am. Phys. Soc., 119, 1997, 12240-12250.

[21] V. L. Bogdanov and V. P. Klochkov "Determination of excited state lifetime of molecules by method of light quenching". Optika i Spektroskopiya, 50, 1981, 1100-1106 (rus.). 